\title{
Efeito da suplementação nutricional com glicina e glutamina, por via oral, na cicatrização colônica em coelhos
}

\author{
Effect of the nutritional supplementation with glicine and glutamine, oral \\ feeding, in the colonic cicatrization in rabbits
}

\author{
Juliano Alves Figueiredo'; Andy Petroianu, TCBC-MG²; Maria Isabel Toulson Davisson Correia, TCBC-MG³ ; Hilário Antônio de \\ Castro Júnior ${ }^{4}$; Wagner Speschit ${ }^{5}$; Raphael de Oliveira Pessoa Silveira ${ }^{6}$; Cristiana Buzelin Nunes ${ }^{7}$; Marcelo Militão Abrantes ${ }^{8}$
}

\section{R E S U M O}

\begin{abstract}
Objetivo: Avaliar o efeito de dois aminoácidos, glicina e glutamina, na epitelização da mucosa colônica, tamanho da área de cicatriz, fibroplasia e resistência tênsil da anastomose intestinal, após colectomia parcial e anastomose término-terminal. Métodos: Quarenta e cinco coelhos adultos, com idade entre 24 e 28 semanas, do sexo masculino, com peso inicial médio de $2.362 \mathrm{~g}$, foram distribuídos aleatoriamente, de forma dupla desconhecida, em cinco grupos, de acordo com o tipo de suplemento. Dois animais morreram em cada grupo por causas diversas. Os grupos de coelhos foram assim distribuídos: Grupo 1 - $(n=9)$ receberam suplementação alimentar com glutamina durante sete dias antes e cinco dias após a operação; Grupo 2 - (n = 9) receberam suplementação alimentar com glicina durante sete dias antes e cinco dias após a operação; Grupo 3 - $(n=9)$ receberam suplementação alimentar com glutamina durante cinco dias após a operação; Grupo 4 - $(n=9)$ receberam suplementação alimentar com glicina durante cinco dias após a operação; Grupo 5 - $(n=9)$ não receberam suplementação alimentar. A resistência da anastomose foi medida por meio de pressão de ruptura por insuflação de ar intraluminar. Resultados: O Grupo 2 foi o único grupo que apresentou valores de ruptura superiores aos do Grupo 5 ( $p<0,05)$. Não houve diferença entre os grupos quanto aos aspectos histológicos estudados. Conclusão: A suplementação oral com glicina, no período pré e pós-operatório, aumenta a resistência tênsil anastomótica colônica após colectomia parcial em coelhos.
\end{abstract}

Descritores: Glicina. Glutamina. Anastomose cirúrgica. Cicatrização de feridas. Colo/metabolismo.

\section{INTRODUÇÃO}

A falha anastomótica é responsável por aumento da morbidade operatória, da permanência hospitalar e dos custos no tratamento cirúrgico das doenças do intestino grosso $0^{1,2}$. Há portanto necessidade de se encontrarem substâncias que melhorem a cicatrização intestinal e que diminuam os índices de complicações.

Somente nas duas últimas décadas, reconheceu-se que a administração de alguns nutrientes, em doses maiores que as necessidades alimentares diárias, pode prevenir deficiências orgânicas e atuar como agente farmacológico ${ }^{3}$. Esses nutrientes podem agir por eles mesmos ou interagir com outros medicamentos. Nutrição terapêutica é o termo que mais adequadamente expressa o efeito desses nutrientes, mas imunonutrição e nutrição farmacológica são frequentemente usados ${ }^{3}$. Nesse sentido a glicina e a glutamina são consideradas como tais.

A glicina é um aminoácido com estrutura molecular simples, e apresenta amplos efeitos biológicos ${ }^{4}$. Utilizou-se, muitos anos, a glicina como aminoácido con- trole de outros aminoácidos, por acreditar-se que tinha efeito inerte como agente biológico ${ }^{4}$. No entanto comprovou-se, em experimentos com animais, que a glicina atua como moduladora na cascata inflamatória sistêmica, possui algum benefício na microcirculação ${ }^{5-7}$ e aumenta a contagem de monócitos na anastomose intestinal ${ }^{8}$.

A glutamina é um aminoácido não-essencial, sendo considerada essencial em situações clínicas com demanda aumentada ${ }^{9}$, tais como: procedimentos cirúrgicos, câncer e sepse. A suplementação de glutamina enteral ou parenteral resulta em aumento da espessura e do aumento do volume protéico da mucosa intestinal ${ }^{10}$. Durante o estado catabólico, a glutamina é importante para o intestino, pois os enterócitos e as células do tecido linfóide usam esse aminoácido como substrato energético. Alguns estudos avaliaram a influência da glutamina na anastomose intestinal ${ }^{8,11,12}$. Um trabalho mostrou que não havia nenhum benefício da suplementação parenteral de glutamina na anastomose intestinal em ratos mal-nutridos. Por outro lado, em dois estudos, os autores encontraram benefício da glutamina oral na anastomoses intestinal de ratos ${ }^{8,12}$.

Trabalho realizado no Departamento de Cirurgia da Faculdade de Medicina da Universidade Federal de Minas Gerais, Belo Horizonte, MG, Brasil. 1. Professor substituto de Cirurgia da Faculdade de Medicina da Universidade Federal de Minas Gerais (FMUFMG), Belo Horizonte, MG, Brasil; 2. TCBC-MG, Professor Titular do Departamento de Cirurgia da FMUFMG, Belo Horizonte, MG, Brasil; 3. Professora Adjunta Doutora do Departamento de Cirurgia da FMUFMG, Belo Horizonte, MG, Brasil; 4. Residente de Urologia do Hospital Felício Rocho, Belo Horizonte, MG, Brasil; 5. Médico; 6. Médico; 7. Mestre em Patologia Clínica pela UFMG, Belo Horizonte, MG, Brasil; 8. Professor da Faculdade de Medicina de Barbacena, Barbacena, MG, Brasil. 
O objetivo deste estudo foi avaliar o impacto da glicina ou da glutamina, administrada por via oral sobre a cicatrização da anastomose colônica, em coelhos.

\section{MÉTODOS}

Quarenta e cinco coelhos adultos, machos, com peso variando entre $2297 \mathrm{~g}$ e $2582 \mathrm{~g}$ foram tratados em gaiolas individuais. Todos os animais tiveram acesso livre a água e à ração. O estudo foi duplo desconhecido. Os animais foram distribuídos em cinco grupos de nove animais. Os animais do Grupo 1 e do Grupo 2 receberam suplementação alimentar com glutamina e glicina 500 mg/ $\mathrm{kg} / \mathrm{dia}$, por sete dias antes da operação e por cinco dias após a operação. Os animais do Grupo 3 e Grupo 4 receberam suplementação somente após a operação, por um período de cinco dias, de cada aminoácido. Os aminoácidos foram diluídos em $5 \mathrm{~mL}$ de água potável, e a administração foi feita por gavagem.

A suplementação nutricional foi realizada em determinado período, de acordo como grupo de coelhos. O Grupo 1 recebeu glutamina por sete dias no pré-operatório e cinco dias no pós-operatório, o Grupo 2 recebeu glicina por sete dias no pré-operatório e cinco dias no pós-operatório. 0 Grupo 3 recebeu glutamina no pós-operatório por cinco dias e o Grupo 4 recebeu glicina no pós-operatório por cinco dias. O Grupo 5 não recebeu suplementação nutricional.

A operação foi realizada após jejum de 15 horas e não houve preparo do intestino. Os animais foram pesados no dia da operação e após seis dias. Os valores de albumina sérica foram mensurados nesses mesmos dias. Sob anestesia com 0,2 mL de xilazina ( $2 \%$ ) e $2,5 \mathrm{~mL}$ de quetamina $(1 \mathrm{~g} /$ $10 \mathrm{~mL}$ ) realizou-se laparotomia mediana. Inicialmente a artéria cólica média foi ligada com fio de seda 5-0 próximo à sua origem na artéria mesentérica cranial. As artérias marginais à direita e à esquerda da cólica média também foram ligadas e seccionadas. Um segmento de cólon transverso de $17 \mathrm{~cm}$ foi removido. A reconstrução do trânsito colônico se fez por meio de anastomose término-terminal com pontos separados seromusculares, utilizando fios de seda 6-0. Após a operação os animais permaneceram em gaiolas individuais, com acesso a água, ração e suplemento, de acordo com o grupo ao qual pertenciam.

Todos os animais foram mortos no $6^{\circ}$ dia do pósoperatório, após anestesia com as mesmas drogas utilizadas na operação. O coelho foi morto por transecção da aorta abdominal.

Para a determinação da pressão de ruptura, um sistema de vasos comunicantes de três vias foi instalado, tendo em uma das vias uma coluna de mercúrio com escala em milímetros. Uma segunda via foi conectada a um compressor de ar (Compressor Schulz, Joinvile, Santa Catarina), com capacidade para $6 \mathrm{~L} / \mathrm{min}$ e calibrado em copo com água, para 150 bolhas / minuto. A terceira via foi introduzida na parte proximal do cólon, a aproximadamente quatro centímetros da anastomose. Distalmente à anastomose, o cólon foi ligado com dois fios de algodão a três centímetros da anastomose.
A medida da pressão foi realizada dentro do abdome, sem que aderência alguma tivesse sido desfeita. A cavidade abdominal foi preenchida com água, a ruptura intestinal foi identificada pela presença de bolhas de ar. O segmento estudado com extensão de $6 \mathrm{~cm}$ apresentava a anastomose em sua porção média. Marcou-se o ponto mais alto que a coluna de mercúrio alcançou sob o efeito da pressão de ar no circuito. Esse valor foi considerado como a pressão de ruptura do cólon ou da anastomose.

O segmento de cólon com a anastomose foi retirado para avaliação histológica. Após abertura longitudinal da borda antimesentérica, o fragmento foi fixado em placa de isopor e mergulhado em formol a $10 \%$, para posterior processamento histológico. Foram utilizadas as colorações por hematoxilina-eosina e tricrômio de Masson, para avaliar qualitativa e quantitativamente o tecido de granulação e a epitelização da mucosa intestinal. A intensidade da fibroplasia foi classificada em ausente (0), leve (1), moderado (2), acentuada (3).

A comparação entre os grupos foi feita pelos testes Kruskal-wallis e Student-Newman-Keusl. A significância estatística foi definida como p menor que $5 \%$.

\section{RESULTADOS}

Não houve diferença na evolução do peso corporal e da albumina sérica. Somente os animais que receberam glicina no pré e no pós-operatórios requereram maior pressão para ruptura da anastomose $(p<0,05)$ (Tabela 1).

A avaliação histológica, com as colorações hematoxilina-eosina e tricrômio de Masson, mostraram que não houve diferença entre os grupos no que diz respeito à epitelização mucosa da anastomose e ao grau de intensidade da fibroplasia, isto é, tecido de granulação.

Dez animais morreram durante o experimento por diversas causas.

\section{DISCUSSÃO}

A fístula anastomótica é uma complicação pósoperatória grave e que eleva a morbidade dos pacientes.

Tabela 1 - Pressão de ruptura conforme os grupos de coeIhos (média e desvio-padrão).

\begin{tabular}{lcc}
\hline Grupo & \multicolumn{2}{c}{ Pressão de ruptura } \\
\cline { 2 - 3 } & Média & DPM \\
\hline 1 & $161,4+38,6$ \\
2 & $218,6^{*}+50,7$ \\
3 & $127,9+59,4$ \\
4 & $206,4+48,5$ \\
5 & $127,8+82,1$ \\
\hline
\end{tabular}

\section{$* p<0,05$}

Grupo 1 - suplementação com glutamina no pré e pós-operatório.

Grupo 2 - suplementação com glicina no pré e pós-operatório.

Grupo 3 - suplementação com glutamina no pós-operatório.

Grupo 4 - suplementação com glicina no pós-operatório.

Grupo 5 -controle. 
Vários trabalhos avaliaram os aspectos fisiopatológicos relacionados ao processo de cicatrização da anastomose intestinal ${ }^{1,8,11-18}$. O presente trabalho estudou os efeitos da glicina e da glutamina na cicatrização da anastomose colônica. Somente a suplementação oral com glicina no pré e pós-operatório foi associada à maior força de ruptura anastomótica.

A resistência da anastomose intestinal, durante o processo de cicatrização, é influenciada por fatores sistêmicos, como o estado nutricional e a presença de neoplasia, dentre outros. Além desses fatores locais, há os relacionados à técnica operatória, suprimento sanguíneo adequado nas margens anastomosadas, tensão sobre as bordas anastomóticas e presença de abscesso perianastomótico, ${ }^{1,18}$ que interferem no resultado. Neste trabalho, avaliou-se a integridade da anastomose intestinal pela insuflação de ar sem desfazer as aderências envolvendo a anastomose. A pressão de ruptura da anastomose intestinal é bom parâmetro para avaliar-se a integridade da anastomose ${ }^{19}$. A tensão de ruptura é também bom parâmetro para avaliar-se a cicatrização intestinal ${ }^{1}$, em correspondência com aspectos histológico. Este último método de avaliação da cicatrização intestinal consiste em aplicar-se força de tração longitudinal ao intestino e perpendicular à linha de anastomose. Preferiu-se medir a pressão de ruptura dentro do abdome, sem desfazer as aderências intestinais, por ser essa situação parecida à que ocorre no pós-operatório 20,21 .

A suplementação dietética interfere no processo de cicatrização intestinal12. Alguns estudos experimentais avaliaram o efeito da suplementação nutricional com aminoácidos. Em particular, interessa a este estudo a suplementação realizada com os aminoácidos glutamina e glicina sobre a resistência anastomótica intestina| $\left.\right|^{8,11,12}$. A glutamina administrada por via parenteral (infusão com 1,2\%) em ratos desnutridos não esteve relacionada com benefício algum na cicatrização da anastomose colônica ${ }^{11}$. Por outro lado, a suplementação nutricional, por via oral com glutamina, durante sete dias, no pós-operatório de colotomia, melhorou a resistência tênsil da anastomose colônica em ratos ${ }^{12}$.

Os resultados conflitantes podem ser explicados por diferenças na via de administração, nas doses, no número de dias em que se administrou o suplemento e na ação dos aminoácidos. Nesse sentido, Matheson et $a l_{1}{ }^{22}$; mostraram que a glutamina suplementada por via enteral em ratos diminui o fluxo sanguíneo para o trato gastrointestinal, especialmente em intestino delgado $^{22}$. Já a glicina estimulou maior concentração de monócitos na área em cicatrização da anastomose colônica ${ }^{8}$.

Estudo recente ${ }^{23}$, realizado com ratos, indicou que a glicina administrada no peroperatório de transplante intestinal, na dose de $1 \mathrm{~g} / \mathrm{kg}$, melhorou a resposta inflamatória, mantendo parte da estrutura muscular íntegra do intestino transplantado e diminuindo a perda da função motora da musculatura lisa intestinal. A suplementação com glutamina nesse estudo não alterou a cicatrização colônica, já a suplementação nutricional com glicina melhorou a resistência tênsil da anastomose.

Os dados da literatura não trazem subsídios para explicar a ação da glicina na pressão de ruptura da anastomose intestinal. Talvez seja pertinente supor que esse aminoácido tenha mantido a microcirculação na área anastomose e modulado a inflamação local nos dias iniciais após o trauma, reduzindo colagenólise.

São necessários mais estudos para compreenderse a ação dos imunonutrientes, na cicatrização. A uniformização dos métodos de avaliação da pressão de ruptura da anastomose e uniformização da via de administração também é necessária para favorecer esse tipo de linha de pesquisa.

Concluindo, a suplementação nutricional com o aminoácido glicina, no pré e pós-operatório, melhorou a resistência tênsil da anastomose intestinal, após insuflação de ar.

\section{Agradecimentos}

Novartis - pela disponibilidade do aminoácido glutamina. Ajinomoto Interamericana (São Paulo, Brasil) - pela disponibilidade do aminoácido glicina.

Centro de Pós-Graduação da Faculdade de Medicina da UFMG.

Meta - Consultoria em Estatística.

\title{
A B $S$ S T
}

\begin{abstract}
Objective: To evaluate the effect of two amino-acids, glycine and glutamine, in the epithelialization of the colonic mucosa, size of the scar area, fibrous as well as the tensile strength of the intestinal anastomoses, after the removal of $17 \mathrm{~cm}$ of the colon and an end-to-end with interrupted suture. Methods: Forty five male adult rabbits varying between 24 and 28 weeks of age with the starting average weight of 2,362 grams were randomly distributed into five groups, according to their respective type of feeding. Two animals died in each group due to different complications. The rabbits were divided into the following groups, according to the procedures performed on them: Group $1-(n=$ 9) received supplemental feeding with glutamine for seven days before and as five days after the operation; Group $2-(n=9)$ received supplemental feeding with glycine for seven days before and five days after the operation; Group $3-(n=9)$ received supplemental feeding with glutamine for five days after the operation; Group 4 - $(n=9)$ received supplemental feeding with glycine for five days after the operation; Group 5 - $(n=9)$ did not receive any supplemental feeding. The resistance of the anastomoses was evaluated through the means of assay for pressure of rupture during intraluminal air insufflation. Results: Group 2 was the only group that presented rupture values above those of Group 5 ( $p$ < 0.05). There was no difference among the groups concerning the histological aspects. Conclusion: Oral supplementation with glycine, in the pre and post-operative periods increases the tensile strength of colonic anastomoses after partial colectomy in rabbits. The oral supplementation with glutamine did not present detectable effect on the colonic anastomoses in rabbits.
\end{abstract}

Key words: Glycine. Glutamine. Anastomosis, surgical. Wound healing. Colon/metabolism. 


\section{REFERENCIAS}

1. Koruda MJ, Rolandelli RH. Experimental studies on the healing of colonic anastomoses. J Surg Res. 1990; 48(5):504-15.

2. Kanto R, Fagundes DJ. Estudo morfológico comparativo de anastomoses colônicas por invaginação versus em plano único extra-mucoso, em cães. Rev Bras Coloproct. 2005; 25(1):51-9.

3. Alexander JW. Nutritional pharmacology in surgical patients. Am J Surg. 2002; 183(4):349-52.

4. Hall C. Glycine. J Parent Enteral Nutr 1998; 22(6):393-8.

5. Roth E, Zellner M, Wessner B, Strasser E, Manhart N, Oehler R et al. Glycine - an inert amino acid comes alive. Nutrition. 2003 19(9):817-8.

6. Wheeler MD, Ikejema K, Enomoto N, Stacklewitz RF, Seabra V, Zhong $Z$ et al. Glycine: a new anti-inflammatory immunonutrient. Cell Mol Life Sci. 1999; 56(9-10):843-56.

7. Zhong Z, Wheeler MD, Li X, Froh $M$, Schemmer $P$, Yin $M$ et al. LGlycine: a novel antiinflammatory, immunomodulatory, and cytoprotective agent. Curr Opin Clin Nutr Metab Care. 2003, 6(2):229-40.

8. da Costa MA, Campos AC, Coelho JC, de Barros AM, Matsumoto HM. Oral glutamine and the healing of colonic anastomoses in rats. JPEN J Parenter Enteral Nutr. 2003; 27(3):182-5; discussion 185-6.

9. Souba WW, Herskowitz K, Salloum RM, Chen MK, Austgen TR. Gut glutamine metabolism. JPEN J Parenter Enteral Nutr. 1990; 14(4 Suppl):45S-50S

10. Souba WW. Glutamine: a key substrate for the splanchnic bed. Annu Rev Nutr. 1991; 11:285-308.

11. McCauley R, Platell C, Hall J, McCulloch R. Effects of glutamine infusion on colonic anastomotic strength in the rat. JPEN J Parenter Enteral Nutr. 1991; 15(4):437-9.

12. Demetriades H, Botsios D, Kazantzidou D, Sakkas L, Tsalis K, Manos $\mathrm{K}$ et al. Effect of early postoperative enteral feeding on the healing of colonic anastomoses in rats. Comparison of three different enteral diets. Eur Surg Res. 1999; 31(1):57-63.

13. Petroianu A, Souza SD, Martins SG, Alberti LR, Vasconcelos LS Influência da vitamina $\mathrm{C}$ e da hidrocortisona sobre a tensão anastomótica jejunal em ratos. Acta Cir Bras. 2000; 15(4):215-9.

14. Petroianu A, Cavalcanti AM, Alberti LR. Efeito da hidrocortisona sobre a pressão de ruptura dos diversos segmentos do tubo digestório de camundongo. An Fac Med Univ Fed Pernamb. 2002 47(2):112-5.
15. Petroianu A, Souza SD, Martins SG, Alberti LR. Influência do ácido ascórbico em anastomoses e alças jejunais íntegras de rato. Arq Gastroenterol 2001; 38(1):48-52.

16. Arantes VN, Okawa RY, Silva AAD A, Barbosa AJA, Petroianu A. Efeito da metilprednisolona sobre a tensão anastomótica jejunal. Arq Gastroenterol. 1994; 31(2):97-102.

17. Pereira WJF, Cavalcanti AMT, Pereira LR, Petroianu A. Resistência das diferentes partes do tubo digestivo em presença ou não de hiperbilirrubinemia. Rev Med Minas Gerais. 2000; 10(1):18-21.

18. Carneiro GT, D'Albuquerque LA, Miranda MP, Scafuri AG, Siva AO. Intestinal anastomoses. Rev Paul Méd. 1992;110(4):183-92

19. Ikeuchi D, Onodera H, Aung T, Kan S, Kawamoto K, Imamura M et al. Correlation of tensile strength with bursting pressure in the evaluation of intestinal anastomosis. Dig Surg. 1999; 16(6):478-85.

20. Del Rio JV, Beck DE, Opelka FG. Chronic perioperative steroids and colonic anastomotic healing in rats. J Surg Res. 1996; 66(2):13842.

21. Mochizuki M, Wu FC, Coy CS, Ayrizono ML, Goes JR, Fagundes JJ. Efeito de aderências dirigidas em anastomoses cólicas isquêmicas em ratos. Acta Cir Bras. 2005; 20(3):247-51.

22. Matheson PJ, Harris BT, Hurt RT, Zakaria ER, Garrison RN. Enteral glutamine supplementation impairs intestinal blood flow in rats. Am J Surg. 2008; 196(2):293-9.

23. Schaefer N, Tahara K, Schuchtrup S, Websky MV, Overhaus M, Schmidt J et al. Perioperative glycine treatment attenuates ischemia/reperfusion injury and ameliorates smooth muscle dysfunction in intestinal transplantation. Transplantation. 2008; 85(9):1300-10

Recebido em 11/09/2008

Aceito para publicação em 14/11/2008

Conflito de interesses: nenhum

Fonte de financiamento: nenhuma

\section{Como citar este artigo:}

Figueiredo JA, Petroianu A, Correia MIT, Castro Júnior HA, Speschit W, Silveira ROP, Nunes CB, Abrantes MM. Efeito da suplementação nutricional com glicina e glutamina, por via oral, na cicatrização colônica em coelhos. Rev Col Bras Cir. [periódico na Internet] 2009; 36(2). Disponível em URL: http://www.scielo.br/rcbc

\section{Endereço para correspondência:}

Andy Petroianu

petroian@medicina.ufmg.br 